\title{
Acceleration of reflex maturation and physical development in suckling rats: effects of a maternal diet containing lipids from goat milk
}

\author{
Juliana Kessia Barbosa Soares, Rita de Cássia Ramos do Egypto Queiroga, \\ Marco Aurélio Delmondes Bomfim, Debora Catarine Nepomuceno de \\ Pontes Pessoa, Elida de Andrade Barbosa, Dione Lins Souza, Jose \\ Eulálio Cabral-Filho, Maria do Carmo Medeiros
}

UFPE, RECIFE, Pernambuco, Brazil

\begin{abstract}
Objective: To investigate the effects of lipids from goat milk containing conjugated linoleic acids on body weight and reflex ontogeny of neonatal rats treated during the prenatal and suckling periods.

Methods: Three groups were studied: soybean oil (S), coconut oil (C), and goat milk lipids (GM). Reflex maturation (palm grasp, righting reflex, cliff avoidance, vibrissae placing, negative geotaxis, auditory startle, and free-fall righting) as well as body weight evolution were recorded during lactation.

Results: Data demonstrated that the lipids from goat milk accelerated body weight evolution as well as all the reflex maturation investigated $(P<0.05)$.

Discussion: The supply of goat's milk offered to Wistar rats during pregnancy and lactation provided a variety of fatty acids necessary to accelerate the development of offspring.
\end{abstract}

Keywords: Goat milk, Conjugated linoleic acid, Rats, Development

\section{Introduction}

Fatty acids are structural components of cell membranes, serve as a source of cellular energy, and functions as a metabolic reserve. Essential fatty acids are a subset of fatty acids that participate in the formation of hormones and bile salts, and should be an adequate part of a daily diet. ${ }^{1}$

During pregnancy and lactation, increasing demands of polyunsaturated fatty acids for the development of neonatal maternal nutrition becomes critical for the morphogenetic processes associated with brain function. ${ }^{2,3}$ In humans, these processes depends on the maturation of the central nervous system that begins in the first trimester of pregnancy and persists until the first years of postnatal life. ${ }^{4}$ In rats, this maturation occurs during late gestation and persists throughout lactation. ${ }^{5}$

In the postnatal period, the cerebellum reach its peak of development resulting of a number of events including: neuronal and glial proliferation, establishment of synaptic contacts, as well as myelination. ${ }^{6}$

Correspondence to: Juliana Kessia Barbosa Soares, UFPE, RECIFE, Pernambuco, Brazil. Email: julianakessia@hotmail.com
The vestibulospinal system is directly related with structures of motor behavior and it has a strong influence on posture during locomotion. ${ }^{7}$ The reflexes ontogeny involves a maturation of the visual, auditory, and motor system. ${ }^{8}$

At this stage, any stimulus in the environment or the organism itself can strongly alter the sequential ontogenetic events, with varied effects on the formation of the nervous system. ${ }^{5,9}$ During this early period of life there is rapid growth of the central nervous system (CNS), the so-called 'critical period' of development and increased vulnerability of the brain. ${ }^{8}$

Thus, the quality and quantity of fatty acids present in the maternal diet are of fundamental importance for the offspring's developing nervous system. Studies involving reflex development and conjugated linoleic acids (CLA) are not found in the literature. CLA is a group of linoleic acids consisting of many different geometrical isomers. ${ }^{10,11}$

In the human diet, CLA is consumed through milk fat and meats derived from ruminant animals, representing $0.5-2 \%$ of the fatty acids present in these foods. ${ }^{12}$ There is evidence indicating that increased consumption of CLA isomers helps reduce body 
weight in humans ${ }^{13}$ and rats. ${ }^{14}$ Analyzing breast milk from women receiving supplementation with CLA was observed in the milk the presence of CLA isomers but there were no modifications in the macronutrients composition. ${ }^{15,16}$ Preliminary studies showed that dietary CLA offered to pregnancy and/or lactating rats are able to crossing the blood-brain barrier of the offspring and stay until a postnatal period. ${ }^{17}$

However, it has been observed that an outcome of drinking goat milk (which CLA is included) versus cow's milk increased weight gain, length, and skeletal mineralization in rats. ${ }^{18}$ Other benefits included the recovery of malabsorption syndrome, by stimulating the absorption of zinc and iron, and preventing anemia. ${ }^{19}$ In addition, consumption of goat milk reduces cholesterol levels without affecting triglycerides and high-density lipoprotein. ${ }^{20}$

The administration of CLA through dietary supplementation decreases angiogenesis ${ }^{21}$ and prostaglandins, with consequent reduction in oxidative damage. ${ }^{22}$ Furthermore, consumption of CLA promotes its incorporation into brain tissue ${ }^{23}$ and protection against toxicity in cortical neurons. ${ }^{24}$

The objective of this research was to investigate whether a maternal diet (containing goat milk fat) offered during the critical period of brain development in rats can interfere in the reflex maturation and body weight gain of offspring. Being so, the present research could in the future support the understanding of the nutritional consequences for children whose mothers are fed this type of diet.

\section{Materials and methods Animals}

Fifty-four Wistar newborn rats from the Department of Nutrition, Federal University of Pernambuco (UFPE), Brazil were used. To obtain these animals, females aged between 120 to 150 days and weighing $250 \pm 50 \mathrm{~g}$ were mated. The pregnancy was confirmed by the presence of sperm in vaginal smear. The pregnant rats were housed in individual polypropylene cages $(60 \times 50 \times 22 \mathrm{~cm})$, in standard conditions with a temperature of $22 \pm 1^{\circ} \mathrm{C}$, a light-dark cycle $(12$ hour light phase beginning at 06:00 hours), and a relative humidity of $65 \pm 5 \%$. The animals received the experimental diets and water ad libitum from the first day of pregnancy until the end of lactation. Standardized litters were randomly assigned six male pups, and weaning was performed at 21 days postnatal. When necessary, female rats were included in the litters to complete six pups, but they were not used in the tests. When the rats of the same group gave birth, the newly born individuals were put together with the other ones who had been born on the same day and after that they were selected at random to form the groups. In this way, the genetic factor did not interfere with the experimental results.

Neonates were weighed every 3 days, from the first day of life until weaning. The experimental protocol followed the recommendations of the National Institutes of Health, USA, and was reviewed and approved by the Ethics Committee on Animal Research Laboratory of Pharmaceutical Technology, Federal University of Paraíba, Brazil (Protocol 0210/07).

\section{Diets}

Three groups of rats were formed according to the respective types of dietary lipids: soybean group (S) - diet containing 7\% of soybean oil (good balance of essential fatty acids) $(n=18)$; coconut group (C) diet containing $7 \%$ of coconut oil (deficient in essential fatty acids) $(n=12)$, and goat milk group (GM) - diet with $7 \%$ of goat' milk fat (source of CLA) $(n=24)$. Thus, only the quality of the fat was changed between diets (Tables 1 and 2).

All diet components were purchased from Rhoster (Sao Paulo, Brazil); in the case of the goat milk diet,

Table 1 Composition of experimental diets

\begin{tabular}{lccc}
\hline & \multicolumn{3}{c}{ Groups } \\
\cline { 2 - 4 } Ingredients $(\mathbf{g} / \mathbf{k g})$ & Soybean & Coconut & Goat milk lipids \\
\hline Cornstarch & 540.0 & 540.0 & 540.0 \\
Sucrose & 100.0 & 100.0 & 100.0 \\
Casein & 190.0 & 190.0 & 190.0 \\
Soybean oil & 70.0 & 0.0 & 0.0 \\
Coconut oil & 0.0 & 70.0 & 0.0 \\
Goat milk lipids & 0.0 & 0.0 & 70.0 \\
Fiber & 50.0 & 50.0 & 50.0 \\
Minerals mix & 35.0 & 35.0 & 35.0 \\
Vitamin mix & 10.0 & 10.0 & 10.0 \\
L-cystine & 3.0 & 3.0 & 3.0 \\
Choline bitartrate & 2.5 & 2.5 & 2.5 \\
Tert-butylhydroquinone & 0.014 & 0.014 & 0.014 \\
\hline
\end{tabular}

Table 2 Fatty acid profile of the different dietary fat source

\begin{tabular}{lccc}
\hline Fatty acids & Soybean oil & Coconut oil & Goat milk lipids \\
\hline C6:0 & 0 & 0 & 0.72 \\
C8:0 & 0 & 0 & 1.33 \\
C10:0 & 0 & 0 & 3.95 \\
C12:0 & 0 & 47.92 & 2.63 \\
C14:0 & 0 & 14.83 & 7.34 \\
C14:1 & 0 & 0 & 0.8 \\
C15:1 & 0 & 0 & 21.55 \\
C16:0 & 2.81 & 9.34 & 22.25 \\
C16:1 & 0 & 0 & 0.37 \\
C17:0 & 0 & 0 & 0.45 \\
C18:0 & 1.12 & 4.25 & 18.06 \\
C18:1n9t & 0 & 0 & 4.11 \\
C18:1n9c & 6.13 & 19.2 & 26.47 \\
C18:2n6c & 15.11 & 4.45 & 3.88 \\
C18:2 ${ }^{*}$ CLA & 0 & 0 & 1.01 \\
C18:3n3 & 74.83 & 0 & 2.35 \\
\hline
\end{tabular}


skimmed milk was immediately cooled and used to prepare the experimental diet. All diets were isocaloric and formulated based on the recommendations of the American Institute of Nutrition (AIN-93G). ${ }^{25}$

\section{Maternal food consumption}

The maternal diet consumption was measured on the gestation days 7, 14 and 21, and in the lactation period at 7,14 and 21 days.

\section{Reflex ontogeny}

Reflexes ontogeny, described below, followed the experimental model established by Smart and Dobbing [8] in rats. Were investigated the followings reflexes:

Palmar grasp $(P G)$ - This reflex consists of a flexion of the digits in response to the stimulation of the handpalm with a small metallic stick. For this reflex, the disappearance date is registered.

Righting $(R)$ - The pup is placed on its back on a flat surface and the expected mature response is to turn over on the ventral surface, resting in the normal position with the four feet on the ground.

Vibrissa placing $(V P)$ - The newborn is held by the tail, with the head facing the edge of a table and the vibrissae just touching the vertical surface of the table. The expected response is to lift the head and to extend the fore legs in direction of the table.

Cliff avoidance ( $C A)$ - The rat is placed on the edge of a 'cliff' (for instance, on the edge of a table), with the forepaws and face just over the edge. The expected response is to move away from the cliff, to avoid dropping.

Negative geotaxis $(N G)$ - The pup is placed on an inclined ramp ( $45^{\circ}$ slope) with its head pointing to the ground. The expected mature response is to turn around and crawl up the slope.

Auditory startle $(A S)$ - The rat is exposed suddenly to a loud, sharp noise. The expected response is a prompt extension of the head and the limbs, followed by withdrawal of the limbs and a crouching posture.

Free-fall righting $(F R)$ - The pup is held with the back downwards $35 \mathrm{~cm}$ above a cotton pad and dropped. The expected response is to turn in mid-air to land on its four paws.

The presence of each reflex was assessed daily between 12:00 and 14:00 hours, from 1 to 21 days postnatal. The first of a series of three consecutive days in which the response was present was considered to be the day of the consolidation of the reflex. A reflex response was considered within a maximum observation period of 10 seconds.

\section{Statistical analysis}

Statistical analysis of the reflex ontogeny was performed using a Kruskal-Wallis test to compare groups followed by Dunn's multiple comparison test.
Table 3 Reflex maturation (median days) of offspring whose mothers were treated with $7 \%$ soybean oil $(n=18)$, coconut oil $(n=12)$ or goat milk $(n=24)$ during pregnancy and lactation

\begin{tabular}{lccc}
\hline Groups & Soybean oil & Coconut oil & $\begin{array}{c}\text { Goat milk } \\
\text { lipids }\end{array}$ \\
\hline Reflex maturation & & & \\
Palm grasp & $6.5(5-9)$ & $9(8-11)$ & $4(3-8)^{\star, \star *}$ \\
Righting reflex & $4(3-6)$ & $5.5(2-10)$ & $3(1-6)^{\star, * \star}$ \\
Cliff avoidance & $6(3-9)$ & $6(2-9)$ & $4(1-6)^{\star, * \star}$ \\
Vibrissae placing & $9.5(8-11)$ & $10(7-11)$ & $9(6-11)^{\star *}$ \\
Negative & $14(7-11)$ & $14(8-14)$ & $10(7-12)^{*, * \star}$ \\
$\quad$ geotaxis & & & \\
Auditory startle & $12(11-13)$ & $13(12-15)$ & $10.5(9-13)^{*, * *}$ \\
Free-fall righting & $18(15-20)$ & $17(14-18)$ & $16(13-17)^{\star}$ \\
\hline
\end{tabular}

The data are shown as median of day of life (minimum and maximum).

Kruskal-Wallis analysis of variance fallowed by Dunn's test ${ }^{*} P<0.05$ versus soybean oil group.

${ }^{*} P<0.05$ versus coconut oil group.Author Queries

One-way analysis of variance followed by Tukey's post hoc test was performed for comparison of weight. The alpha error to reject the null hypothesis was $P<0.05$.

\section{Results}

As shown in Table 3, the offspring of mothers treated with goat milk fat during pregnancy and lactation compared to control offspring (S) showed an anticipation in the disappearance of $\mathrm{PP}$ and the appearance of the following repercussions: PR, CA, NG, AS, FR $(P<0.05)$ for all groups). The same group (GM) compared with coconut oil (C) showed similar effects for the following reflexes: PP, PR, AC, VP, NG, AS. There was no significant difference between groups $C$ and $\mathrm{S}$ for the following reflexes: PG, RR, CA, VP, NG, RS, FR $(P<0.05)$ for all groups).

The body weight of the GM group was significantly higher from the third day of life compared to animals treated with soybean oil (S) or coconut oil (C), these differences persisted until the end of lactation (Fig. 1). On the first day of life the body weight of the GM group was higher than the coconut group.

Maternal food intake was increased in the $\mathrm{C}$ and GM compared to $\mathrm{S}$ group only on day 21 of gestation $(P<0.05)$. The feed intake is presented in Fig. 2.

\section{Discussion}

The results of this study indicate that a maternal lipids diet containing fat from goat milk can affect the reflex ontogeny and body weight of offspring compared to controls (soybean and coconut oil). Lipids from goat milk, which were offered to pregnant and lactating rats, contain saturated, polyunsaturated fatty acids and CLA in their lipid composition. CLA was found only in the GM group. The predominant fatty acids found in the soybean oil containing diet control group were polyunsaturated (linoleic acid and linolenic 


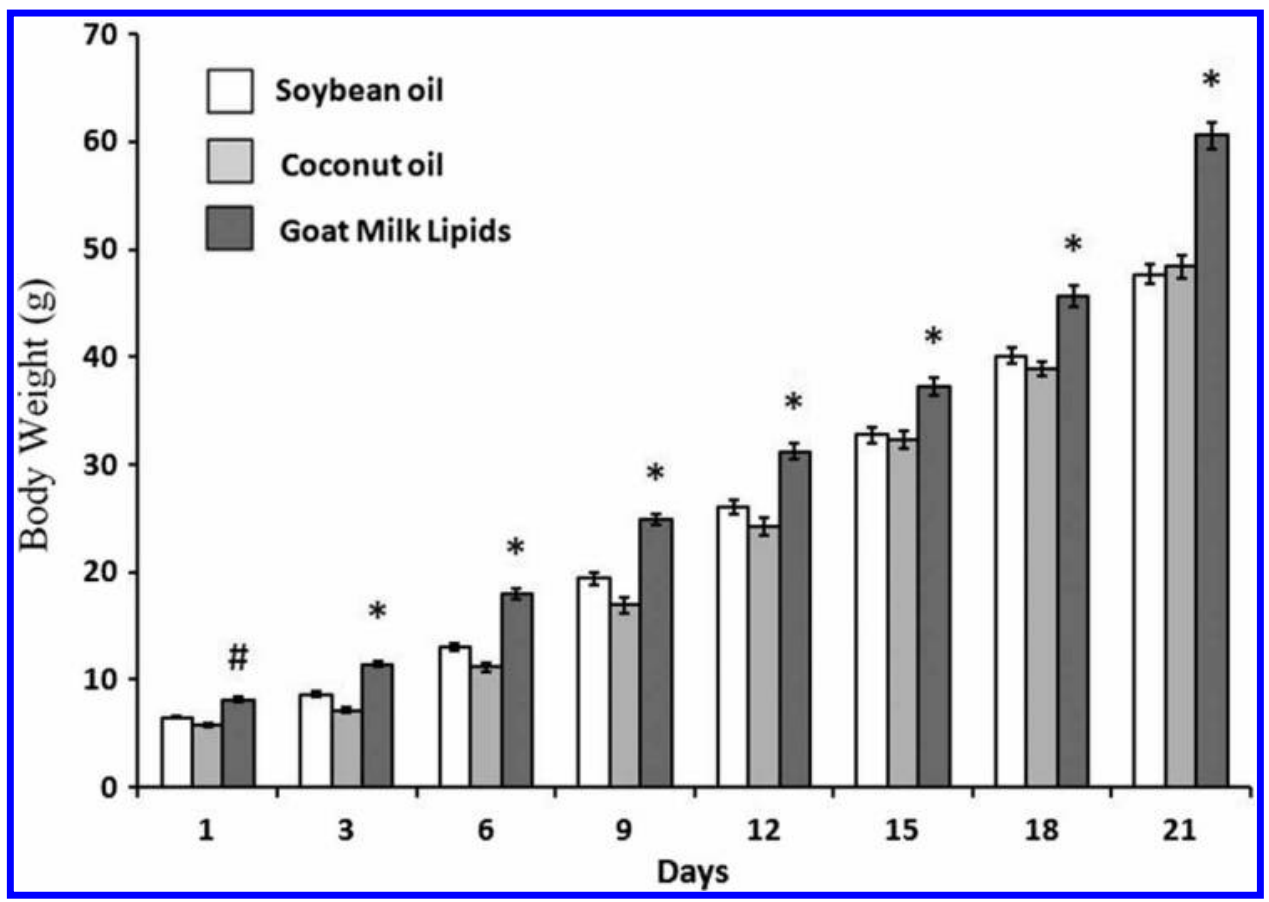

Figure 1 Mean body weight in grams (g) $( \pm$ SEM), of rat infants whose mothers received a diet containing $7 \%$ soybean oil $(n=18)$, coconut oil $(n=12)$ or goat milk lipids $(n=24)$ during pregnancy and lactation. Kruskal-Wallis analysis of variance followed by Dunn's test was performed. $P<0.05$, (*) a statistically significant change compared to the soybean oil group, (\#) a statistically significant change compared to the coconut oil group.

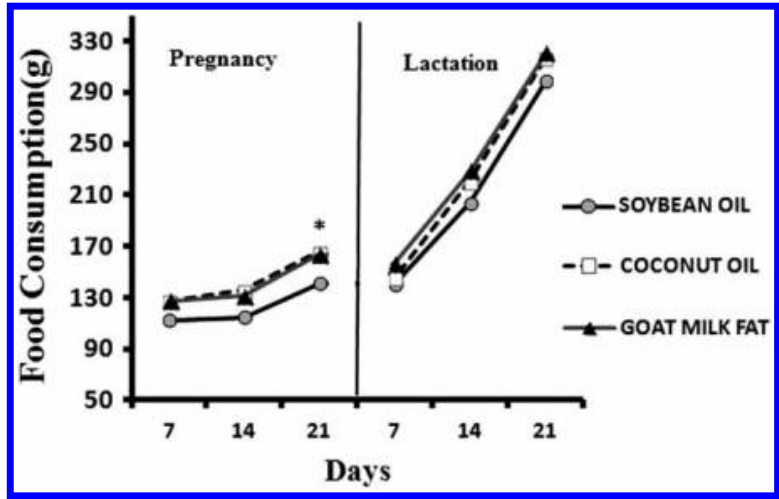

Figure 2 Weekly means of maternal food intake (g) for each experimental group during pregnancy and lactation. The diets are similar except for the fat source: soybean oil, coconut oil, and goat milk fat source of CLA. *Statistically significant compared to soybean group $(P<0.05)$.

acid) whereas saturated fatty acids such as lauric acid and myristic acid were found in the $\mathrm{C}$ group.

Based on these results, we conjecture that beyond the duration of dietary treatment, the profile of fatty acids and especially the presence of CLA can induce specific effects on reflex ontogeny.

In a previous study, rats that were fed a diet supplemented with soybean and sunflower oil containing polyunsaturated fatty acids during pregnancy and lactation also showed an acceleration of several reflexes. ${ }^{26}$ A similar result was also observed by Gozzo and Oliverio, ${ }^{27}$ in which animals were treated with a diet containing odd chain fatty acids. These results demonstrate that changes in maternal dietary fatty acids can anticipate reflex ontogeny in the offspring, which are similar to the results found in this study.

Dietary sources of essential fatty acids, including myristic, palmitoleic, stearic, and oleic, were associated with increased mRNA levels of proteolipid protein and myelin oligodendrocyte glycoprotein, which is involved in the synthesis of myelin in the brain of newborn rats. ${ }^{28}$ According to the authors, the mediators responsible for the increased synthesis of myelin are also associated with accelerated maturation reflex. These lipids may be a mechanism linking diet and neural maturation. This is a hypothesis that should be tested in future.

During pregnancy, the metabolic demands for fatty acids in the brain is high for mothers and their fetuses. ${ }^{2,28}$ It has also been shown that in mouse embryos between 14 and 17 days old, there is an increased concentration of polyunsaturated fatty acids in the brain. ${ }^{29}$ Thus, taking into account the importance of fatty acids during the rapid development of the nervous system, ${ }^{30-32}$ it is possible that the observed changes in ontogeny reflex may be associated with a possible change in the accumulation of these fatty acids in the brains of newborns during this period.

The aforementioned studies demonstrate the effects of various lipids to the developing central nervous system. There is evidence suggesting that after CLA supplementation, CLA can be incorporated in different organs, including the brain, that this fatty acid has the ability to cross the blood-brain barrier. ${ }^{23,33}$ 
Furthermore, CLA acts on the nervous system by protecting neurons from toxic agents. ${ }^{24}$ Therefore, the presence of CLA, along with a variety of fatty acids found in goat milk can contribute to changes in the maturation of the nervous system, as revealed by an early developmental requirement shown in this study. Moreover, previous findings have shown that ketogenic diets using animal lipids ${ }^{34}$ and a multi-deficient diet $^{35}$ retard the developmental reflex. Other studies have also identified delayed maturation of reflexes using diets containing high levels of omega-3. ${ }^{36,37}$ Based on the above findings, we can see that both the quality and quantity of dietary lipids may affect the appearance of reflexes.

In the present study, we observed an increase in the body weight of newborn rats treated with lipid goat milk compared to the control group (S) and group treated with coconut oil (C). Some studies have demonstrated the influence of CLA on the body weight of rats. ${ }^{38}$ Results similar to ours were observed by others in which the effects of CLA supplementation on the development and growth of rats during pregnancy and/or lactation were investigated and an increase in weight gain of pups during the postnatal period (10 days of lactation) was observed. ${ }^{39}$ Moreover, the ketogenic diet resulted in a reduction in the pup's body weight. ${ }^{40}$ Borba et al. ${ }^{41}$ using a diet of coconut oil $(5 \%)$ for two successive generations did not observe a reduction in the body weight of offspring in the first generation; however, reduced body weight in the second generation was observed.

These data reinforce the importance of dietary lipids to the developing body and nervous system. In accordance with the foregoing, findings, the assumption is that changes in lipid composition of the diet may alter the reflections of development and maturation. Changes in the quality and quantity of lipids interfere directly with chronology of reflex response, as observed in this study.

\section{Conclusion}

Our results demonstrate the impact of lipids in goat milk, a source of CLA on the early maturation reflexes of rats. Also evident in our study was the increase in body weight of offspring treated with goat milk. These results reinforce the role of dietary lipids in neurodevelopment. To evaluate the effects associated with these results, other studies should be conducted to elucidate what dietary lipids are beneficial during early maturation and which mechanisms are involved in this process.

\section{Acknowledgements}

The authors thank the financial support from the Brazilian agencies Embrapa, CAPES, and CNPq.

\section{References}

1 Valenzuela AB, Nieto SK. Ácidos grasos omega-6 y omega-3 en la nutrición perinatal: su importância em el desarrolo del sistema nervioso y visual. Rev Chil Pediatr 2003;74:149-57.

2 Koletzko B, Rodriguez-Palmero A, Demmelmair H, et al. Physiological aspects of human milk lipids. Early Hum Dev 2001;65:S3-18.

3 Xiang M, Alfven G, Blennow M, Trygg M, et al. Long-chain polyunsaturated fatty acids in human milk and brain growth during early infancy. Acta Paediatr 1999;89:142-7.

4 Martinez M. Tissue levels of polyunsaturated fatty acids during early human development. J Pediatr 1992;120:S129-38.

$\checkmark 5$ Morgane PJ, Miller M, Kempler T, et al. Prenatal malnutrition and development of the brain. Neurosc Behav Rev 1993;17: 91-128.

6 Altman J, Bayer SA. Development of the cerebellar system in relation to its evolution, structure and functions. In: The central nervous system, structure and function, New York, 1997, pp. 1-783.

7 Boyle R. The vestibular labyrinth in health and disease. In: Vestibulospinal control of reflex and voluntary head movement. Annals of the New York Academy of Sciences, New York, 2001, pp. $364-80$

-8 Smart JL, Dobbing J. Vulnerability of developing brain. II. Effects of early nutritional deprivation on reflex ontogeny and development of behavior in the rat. Brain Res 1971;28:85-95.

$\checkmark 9$ Dobbing J. Undernutrition and the developing brain. The relevance of animal models to the human problem. Am J Dis Child 1970;120:411-5.

10 Banni S. Conjugated linoleic acid metabolism. Curr Opin Lipidol 2002;13:261-6.

11 Park Y. Conjugated linoleic acid (CLA): Good or bad trans fat? J Food Compos Anal 2009;22S:S4-S12.

-12 McLeod RS, Leblanc AM, Langille MA, Mitchell PL, Currie DL. Conjugated linoleic acids, atherosclerosis, and hepatic very-low-density lipoprotein metabolism. Am J Clin Nutr 2004;79:1169S-74S.

13 Wang YM, Jones PJH. Conjugated linoleic acid and obesity control: efficacy and mechanisms. Int J Obesity 2004; 28:941-55.

14 Wang YM, Nagao K, Inoue N, et al. Isomer-specific anti-obese and hypolipidemic properties of conjugated linoleic acid in obese OLETF rats. Biosci Biotechnol Biochem 2006;70:355-62.

15 Hasin A, Griinari JM, Williams JE, et al. Consumption of c9,t11-18:2 or t10,c12-18:2 enriched dietary supplements does not influence milk macronutrients in healthy, lactating women. Lipids 2007;42:835-43.

16 Mosley SA, Shahin AM, Williams J, et al. Supplemental conjugated linoleic acid consumption does not influence milk macronutrient contents in all healthy lactating women. Lipids 2007;42: 723-9.

17 Soares JKB, Rocha-de-Melo AP, Medeiros MC, et al. Conjugated linoleic acid in the maternal diet differentially enhances growth and cortical spreading depression in the rat progeny. Bioch Biophy Acta 2012;1820:1490-5.

18 Park YW, Mahoney AW, Hendricks DG. Bioavailability of iron in goat milk compared with cow milk fed to anemic rats. J Dairy Sci 1986;69:2608-15.

19 Alferez MJM, Lopez-Aliaga I, Nestares T, et al. Dietary goat milk improves iron bioavailability in rats with induced ferropenic anemia in comparison with cow milk. I Dairy J 2006;16: 813-21.

-20 Lopez-Aliaga MJM, Alferez MT, Nestares PB, et al. Goat milk feeding causes an increase in biliary secretion of cholesterol and a decrease in plasma cholesterol levels in rats. J Dairy Sci 2005;88: 1024-30.

21 Sikorski AM, Hebert N, Swain RA. Conjugated linoleic acid (CLA) inhibits new vessel growth in the mammalian brain. Brain Res 2008;1213:35-44.

22 Nakanishi T, Koutoku T, Kawahara S, et al. Dietary conjugated linoleic acid reduces cerebral prostaglandin E2 in mice. Neurosc Letters 2003;341:135-8.

23 Alasnier C, Berdeaux O, Chardigny JM, et al. Fatty acid composition and conjugated linoleic acid content of different tissues in rats fed individual conjugated linoleic acid isomers given as triacylglycerols. J Nutr Biochem 2002;13:337-45.

24 Hunt WT, Kamboj A, Anderson HD, et al. Protection of cortical neurons from excitotoxicity by conjugated linoleic acid. J Neurochem 2010;115:123-30. 
25 Reeves PG, Nielsen FHC, Fahey GC. AIN-93 purified diets for laboratory rodents: final report of the American Institute of Nutrition. J Nutr 1993;123:939-51.

-26 Santillan ME, Vincenti LM, Martini AC, et al. Developmental and neurobehavioral effects of perinatal exposure to diets with different n-6:n-3 ratios in mice. Nutrition 2010;26:423-31.

$\checkmark 27$ Gozzo S, Oliverio A. Effects of dietary phospholipids and oddchain fatty acids on the behavioural maturation of mice. Food Chem Toxic 1982;20:153-7.

28 Salvati S, Sanchez M, Campeggi LM, Suchanek G, Breitschop $\mathrm{H}$, Lassmann H. Accelerated myelinogenesis by dietary lipids in rat brain. J Neurochem 1996;67(4):1744-50.

29 Green P, Glozman S, Kamensky B, et al. Developmental changes in rat brain membrane lipids and fatty acids. The preferential prenatal accumulation of docosahexaenoic acid. J Lipid Res 1999;40:960-6.

30 Bourre JM. Effects of nutrients (in food) on the structure and function of the nervous system: update on dietary requirements for brain. Part 2: macronutrients. J Nutr Health Aging 2006; 10:386-99.

31 Clandinin MT. Brain development and assessing the supply of polyunsaturated fatty acid. Lipids 1999;34:131-7.

32 Green P, Yavin E. Natural and accelerated docosahexaenoic acid accumulation in the prenatal rat brain. Lipids 1996;31: S235-8.

- 33 Fa M, Diana A, Carta G, et al. Incorporation and metabolism of c9, t11 and t10, c12 conjugated linoleic acid (CLA) isomers in rat brain. Biochim Biophys Acta 2005;1736:61-6.

- 34 Soares AKF, Guerra RGS, Castro ML, et al. Somatic and reflex development in suckling rats: effects of mother treatment with ketogenic diet associated with lack of protein. Nutr Neurosc 2009;12:260-6.

-35 Anselmo CWSF, Santos AAA, Freire CMA, et al. Influence of a $60 \mathrm{~Hz}, 3 \mathrm{~T}$, electromagnetic field on the reflex maturation of wistar rats offspring from mothers fed a Regional Basic Diet during pregnancy. Nutr Neurosc 2006;9:201-6.

- 36 Amuskivar E, Francisco J, Ruperez CB, et al. Low arachidonic acid rather than $\alpha$-tocopherol is responsible for the delayed postnatal development in offspring of rats fed fish oil instead of olive oil during pregnancy and lactation. J Nutr 2000;130:2855-65.

37 Saste MD, Carvier JD, Stockard JE, et al. Maternal diet fatty acid composition affects neurodevelopment in rat pups. J Nutr 1998;128:740-3.

- 38 Hayashi AA, Medeiros SR, Carvalho MH, et al. Conjugated linoleic acid (CLA) effects on pups growth, Milk composition and lipogenic and lipogenic enzymes in lactating rats. J Dairy Res 2007;74:160-6.

-39 Chin SF, Storksoh JM, Albright KJ, et al. Conjugated linoleic acid is a growth factor for rats as shown by enhanced weight gain and improved feed efficiency. J Nutr 1994;124:2344-9.

- 40 Ziegler DR, Araujo E, Rotta LN, et al. A ketogenic diet increases protein phosphorylation in brain slices of rats. J Nutr 2002;132:483-7.

-41 Borba JMC, Rocha-de-Melo AP, Santos AAS, et al. Essential fatty acid deficiency reduces cortical spreading depression propagation in rats: a two-generation study. Nutr Neurosci 2010;13: 144-50.

-42 Herrera E. Implications of dietary fatty acids during pregnancy on placental fetal and postnatal development - a review. Placenta 2002;23:S9-S19. 